\title{
Antreiber evolutionärer Transformation: die Endosymbiose
}

MONA SCHREIBER, SVEN B. GOULD

ZELLBIOLOGIE DER PFLANZEN, UNIVERSITÄT MARBURG

\section{Endosymbiosis is a transformative force of evolution. Endosymbionts} established billions of years ago shaped the face of earth and more recent ones take up intriguing new duties. Benefits of exploring endosymbioses are manyfold: we gain a better understanding of fundamental biological principles such as why prokaryotes fail to frequently evolve eukaryote-like complexity and can learn how beneficial partnerships are established. 50 years ago, endosymbiosis was met with scepticism, but is now accepted as a phenomenon responsible for some of life's biggest transitions.

DOI: $10.1007 / \mathrm{s} 12268-021-1670-9$

(C) Die Autorinnen und Autoren 2021

Charles Darwins Evolutionstheorie fußt auf der Idee der Vererbung kleinster vorteilhafter Veränderungen und deren Fixierung in einer Spezies. So formulierte er, nur auf Beobachtung der Natur beruhend, eines der bedeutendsten Konzepte der theoretischen Biologie und legte das Fundament für unser Verständnis der vertikalen Vererbung adaptiver Eigenschaften. Im Laufe der Zeit führten neue Erkenntnisse, wie die Mendelsche Vererbungslehre, die Entdeckung der DNAMutation und die Populationsgenetik, zu Anpassungen der Evolutionstheorie. In den vergangenen Jahrzehnten gewann die horizontale Vererbung genetischer Information (horizontal gene transfer, HGT) an Bedeutung. Endosymbiose kann als extreme Form von HGT verstanden werden, bei der das Wirtsgenom mit Hunderten Genen und Darwins Theorie um eine vertikale Komponente erweitert werden. Am eindrücklichsten zeigt sich diese transformierende Kraft im Ursprung der Eukaryoten sowie später in der Entwicklung der Archaeplastida.

\section{Zwei Milliarden Jahre bis zum} Ursprung eukaryotischer Zellbiologie Der Übergang von einer Geo- zur ersten Biochemie vor etwa vier Milliarden Jahren markierte den Ursprung des Lebens. Die Erfor-

schung der Abiogenese hat aktuell beachtliche Fortschritte gemacht und so konnte durch den Einsatz von Greigit $\left(\mathrm{Fe}_{3} \mathrm{~S}_{4}\right)$ oder Awaruit $\left(\mathrm{Ni}_{3} \mathrm{Fe}\right)$ - charakteristische Mineralien der Hydrothermalquellen - $\mathrm{CO}_{2}$ zu Acetat und Pyruvat unter der Nutzung von $\mathrm{H}_{2}$ als Elektronendonor reduziert werden [1]. Diese Reaktionen spiegeln den Acetyl-CoA-Syntheseweg wider und damit einen der ältesten biochemischen Synthesewege. Er ist Grundbaustein des ersten Lebens wie der genetische Code, das Ribosom und die etwa 350 universellen Proteinfamilien, welche auf LUCA (last universal common ancestor) zurückgehen. Das erste Leben war in seiner Natur rein prokaryotisch und blieb es für rund zwei Milliarden Jahre auch. Warum?

Die fundamentalen Unterschiede in der Lipidbiochemie der Archaeen und Bakterien belegen die frühe Aufspaltung der beiden Gruppen. Sie beeindrucken in evolvierter Biochemie und Enzymatik, während ihre zelluläre und morphologische Komplexität gering bleiben. Nur vereinzelt finden sich membrangebundene Kompartimente wie die Thylakoide in Cyanobakterien oder das

\section{TSAR}

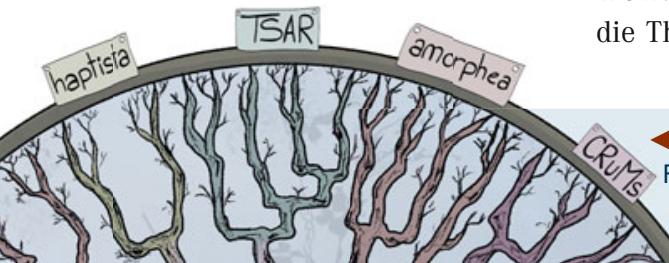

Abb. 1: Der Baum des Lebens mit Fokus auf die Eukaryoten. Der letzte gemeinsame universale Vorfahr (last universal common ancestor, LUCA) spaltete sich vor rund vier Milliarden

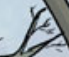
Jahren in Archaeen und Bakterien auf. Die Integration eines Bakteriums in einen archaeelIen Wirt leitete die Eukaryogenese rund zwei Milliarden Jahre später ein und mündete in die Evolution der verschiedenen eukaryotischen Linien. Eine zweite Endosymbiose mit einem Cyanobakterium markierte den Ursprung der Archaeplastida vor mindestens 1,5 Milliarden Jahren, aus welcher Gruppe vor ca. 500 Millionen Jahren eine stroptophytische Alge ihren Weg an Land fand und letztendlich die Embryophyta evolvierten. CRUMs: Collodictyonida, Rigifilida und Mantamonadida;

TSAR: Telonemids, Stramenopila, Alveolata und Rhizarien; Ga: Giga-annum ( $10^{9}$ Jahre). 


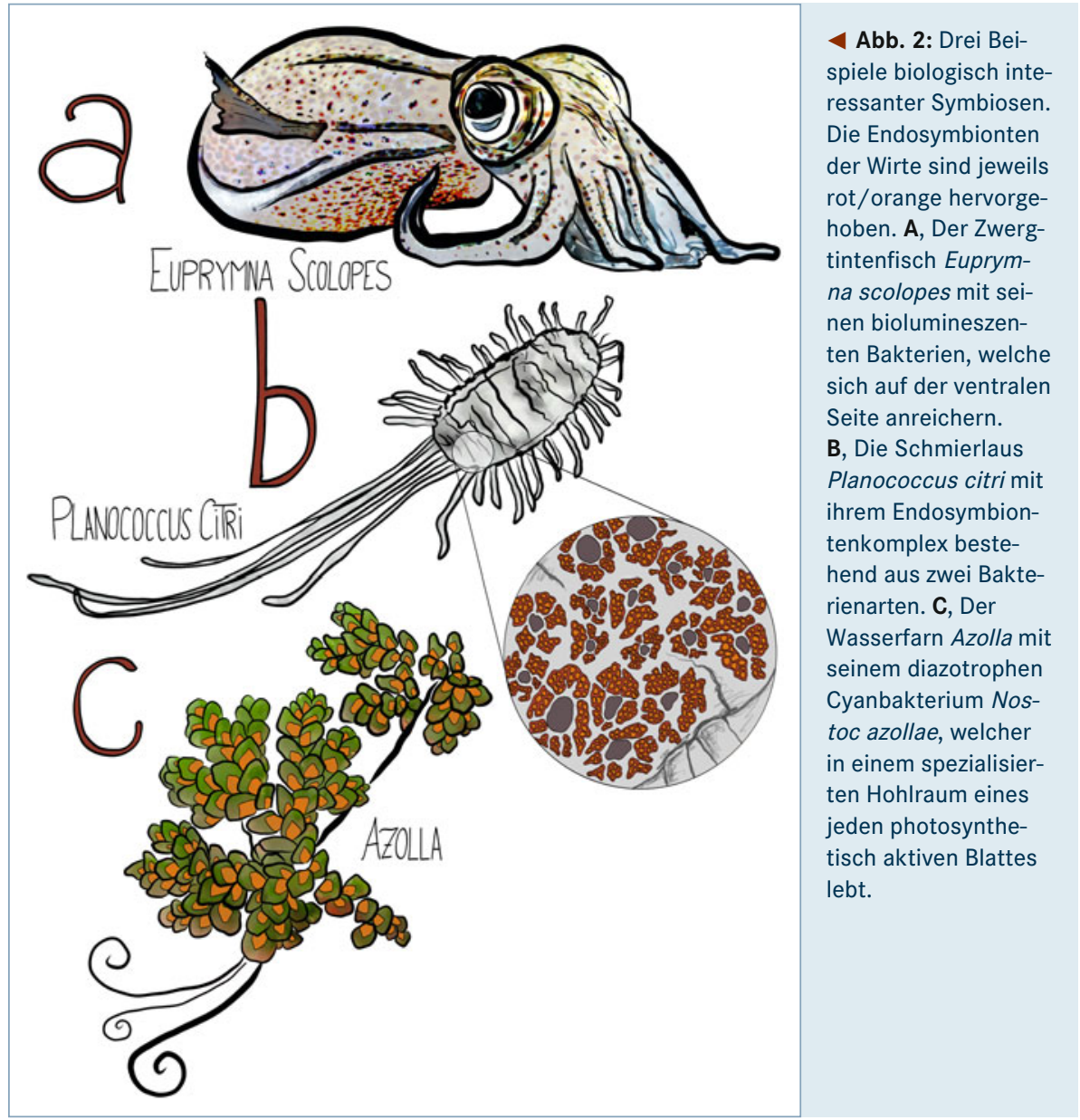

Acidocalcisom in Agrobakterien. Kompartimente sind aber kein definierendes Merkmal der Prokaryoten und ihre Evolution mündete nie in ein Taxon mit komplexem und mehrzelligem Aufbau [2]. Erst die Perfektionierung der syntrophischen Beziehung zwischen einem Archaeon und einem Alphaproteobakterium führten zur eukaryotischen Zellbiologie (Abb. 1).

Phylogenetisch stehen die durch Metagenomdatenassemblierung entdeckten Asgardarchaea der Wirtszelle der Eukaryogenese am nächsten. Vertreter dieser diversen Gruppe codieren eine Reihe von Proteinen, welche als typisch für Eukaryoten und ihre Charakteristika galten: ESCRT-Proteine des Endomembransystems, Aktinzytoskelettregulierende Komponenten und ein Ubiquitinierungssystem beispielweise. Erste Aufnahmen des Asgardarchaeons Prometheoarchaeon syntrophicum zeigen jedoch keinerlei eukaryotische Merkmale und mit nur 0,3 Prozent ist die Menge an Proteinfamilien des last eukaryotic common ancestor (LECA), die exklusiv auf Asgardarchaea zurückgehen, sehr gering. Genomgröße und Anzahl codierter Proteine korrelieren eben nicht mit der Komplexität einer Spezies. So codieren manche prokaryotischen Taxa an die 10.000 Proteine, einige Eukaryoten wie Encephalitozoon hingegen nur 2.000. Die zelluläre Komplexität der Eukaryoten sowie ihre innovative Kraft in der Entwicklung neuer Proteinfamilien gehen auf andere Mechanismen zurück und führen zum Mitochondrium.

In der Eukaryogenese war die Rolle des Mitochondriums vielgestaltig. Es zahlte für die Explosion an Proteinfamilien mit einer optimierten Energieversorgung sowie der Verlagerung seiner genetischen Information und dessen Regulation in den Zellkern. Damit konnte die Proteinbiosynthese, dem mit etwa 70 Prozent des gesamten ATP-Budgets teuersten Prozess, in Eukaryoten um das etwa 200.000-Fache gesteigert werden [3]. Früh ermöglichte dies die Entstehung tausender neuer Proteinfamilien, welche die eukaryotische Zelle stützen und steuern. Neben Energie lieferte das Mitochondrium auch Membranmaterial in Form von sekretierten Vesikeln. Mitochondria-derived vesicles (MDVs) transformierten die Lipidzusammensetzung von innen heraus und bildeten
Kompartimente. Bis heute sind MDVs an der Neusynthese von Peroxisomen beteiligt, fusionieren mit diversen Kompartimenten und unterstützen unser Immunsystem.

Viele der eukaryotischen Kompartimente und Merkmale fungieren als evolutionäre Lösung für die Beherbergung eines Endosymbionten. Die Entstehung von Zellzyklus und sexueller Vermehrung erlaubten die Synchronisation beider Partner, ebenso wie die Rekombination der DNA der Wirtszelle mit der des Endosymbionten, welcher von der Umwelt abgeschnitten und damit Muller's Ratchet ausgeliefert ist (also der irreversiblen Akkumulation von schädlichen Mutationen durch den Verlust der Rekombinationsfähigkeit einer freilebenden Zelle). Es entstanden Autophagosome für den Abbau defekter Endosymbionten sowie Golgi-Apparat und endoplasmatisches Retikulum für die räumliche Trennung wenig spezifischer Proteine der Glykosylierung und der Sortierung von Vesikelfluss. Indirekt bestätigen Haloarchaeen, als mögliches Ergebnis einer gescheiterten Endosymbiose, diese Idee. Sie codieren etwa 1.000 Proteine (inkl. einer funktionellen Elektronentransportkette), welche auf einen konzentrierten HGT bakteriellen Ursprungs zurückgehen, zeigen jedoch keinerlei intrazelluläre Komplexität. Kein Endosymbiont, kein selektiver Druck interne Komplexität zu fixieren und damit eine Reversion hin zu prokaryotischer Biologie und Evolution. Es spricht vieles dafür, dass das Mitochondrium sowohl die energetische Freiheit lieferte, um eukaryotische Biologie zu evolvieren, als auch den Grund diese bis heute zu fixieren.

\section{Die Plastide und der Ursprung eukaryotischer Photosynthese}

Keine eukaryotische Gruppe veränderte das Gesicht der Erde maßgeblicher als die „grüne Linie“. Vor rund 1,5 Milliarden Jahren begann der Siegeszug der Archaeplastida, der monophyletischen Linie, welche Algen und Landpflanzen vereint. Die funktionelle Integration eines Cyanobakteriums in einen heterotrophen Wirt führte zum Ursprung phototropher Eukaryoten. Durch die bereits zuvor erfolgte Integration des Mitochondriums in eine Wirtszelle waren alle nötigen Voraussetzungen für die Transformation eines Cyanobakteriums in eine Plastide vorhanden und dem heterotrophen Protisten wurden keine zusätzlichen Kompartimente hinzugefügt. Stattdessen wurden Kompartimente wie Peroxisom und Vakuole physiolo- 
gisch angepasst und andere wie die Dynamin-unterstützte Teilung der Plastide, die Redoxregulation durch Thioredoxine und das Autophagosom (und dessen Regulation durch Gene der $A T G$-Familie) für die Plastide recycelt und optimiert.

Die hohe Komplexität der erfolgreichen Integration spiegelt sich schon darin wider, dass es keine zweite Eukaryogenese und keine anderen Supergruppen phototropher Eukaryoten gibt. Jede Mutation oder ein falscher Ablauf in der Synchronisation regulatorischer Prozesse, wie denen der retrograden Signalkaskade, können das Ende eines evolutionären Experiments bedeuten. So reichten vermutlich einzelne Punktmutationen in Genen der Phytoen- oder Chlorophyllsynthese aus, um aus mixotrophen Schwestergruppen von Chlamydomonas heterotrophe Taxa zu machen. Für die erfolgreiche Partnerschaft mussten z. B. hunderte prokaryotische Gene in das Genom der Wirtszelle integriert und mit eukaryotischen, regulatorischen Elementen und Sequenzinformationen für die Erkennung durch den Importapparat (translocator of the outer/inner chloroplast membrane, TOC/TIC) versehen werden.

Die Biochemie der Plastide beinhaltet die Synthese von Aminosäuren, Isoprenoiden, Häm, die Speicherung von Stärke und natürlich die oxygene Photosynthese in der Thylakoidmembran. Wie die Atmungskette der Mitochondrien, so generiert die Photosynthese eine bioenergetische Membran, die als Quelle reaktiver Sauerstoffspezies eine recht explosive Biochemie aufweist. Da die Photosynthese nicht abzustellen ist, so lange Lichtenergie einfließt, bedarf es eines hochkomplexen Netzwerks zur Regulation des Elektronenflusses und der nicht photochemischen Fluoreszenzlöschung. Ausschließlich in der grünen Linie, den Chloroplastida (Abb. 1), evolvierte durch die Duplikation von TOC75 ein dynamisches Importsystem, welches eine effiziente und schnelle Antwort auf Lichtstress unterstützt. Für den Landgang der Pflanzen und zur Überwindung der damit verbundenen abiotischen Stressfaktoren war dies ein kritischer Faktor [4].

Vor 500 Millionen Jahren charakterisierte karger Fels die Landmassen, welcher dem Leben kein nennenswertes Substrat bot. Dies änderte sich erst mit dem Landgang einer streptophytischen Alge.
Um die Transition an Land zu ermöglichen, kam es zu zahlreichen Innovationen, wie der Ausbildung einer Cuticula gegen Austrocknung, eines Leitgewebes (Xylem/Phloem) für den vertikalen Transport von Wasser und Nährstoffen, von Stomata zum Gasaustausch, Samenkapseln gegen Austrocknung und Pathogene, von Rhizoiden und Wurzeln, wie auch arbuskulären Symbiosen und Mykorrhiza für eine optimierte Substrataufnahme. Ferner ist die Entkopplung der Plastiden von der Zellkernteilung bedeutsam, da sie zur Entwicklung von Polyplastidie (mehr als zwei Plastiden pro Zelle) führte. Dieses charakteristische Merkmal makroskopischer Pflanzenbaupläne war evolutionäre Voraussetzung für die Zunahme an Biomasse. Der Landgang der Pflanzen generierte Substrat, steigerte die Sauerstoffkonzentration um rund das Zehnfache und ermöglichte (oder zumindest begünstigte) die Kambrische Artenexplosion. Die Grundsubstanz der tierischen, extrazellulären Matrix, das Collagen, entsteht durch Quervernetzung von Procollagen-Untereinheiten durch die Lysyloxidase, welche Sauerstoff abhängig ist. So führte die erste Endosymbiose (Eukaryogenese) zum Ursprung komplexen Lebens und die zweite zum Ursprung makroskopischen Lebens, auch durch die Optimierung eines cyanobakteriellen Endosymbionten und seiner photosynthetischen Leistung.

\section{Endosymbiontischer Spielplatz der Evolution}

Mitochondrium und Plastide sind die prominentesten Beispiele der Endosymbiontentheorie. Doch die Evolution brachte zahlreiche weitere Fälle hervor, unter den Protisten ebenso wie den Metazoen und Pflanzen [5]. Während die Archaeplastida monophyletischen Ursprungs sind, wurde ihre Plastide mehrfach durch sekundäre - und in Dinoflagellaten auch durch tertiäre und quartäre - Endosymbiose weitergereicht. Auf sekundäre Endosymbiose zurückgehende Organismen sind von bedeutendem ökologischem (z. B. Diatomeen als Primärproduzenten) und auch ökonomischen Interesse (z. B. negativer Einfluss durch den Erreger der Malaria). Sie zeigen eindrucksvoll, dass die irreversible Integration eines Eukaryoten in einen anderen einfacher zu sein scheint als die eines Prokaryoten in einen Eukaryoten. 
Schätzungen zufolge tragen rund fünf Prozent aller Algen Endosymbionten und sind selbst häufig (endo)symbiontische Partner, wie bei Paramecium bursaria, der grünen Hydra (Hydra viridis), den Eiern des Querzahnmolchs Ambystoma oder dem RoscoffWurm Symsagittifera.

Ein interessantes Beispiel für biolumineszente, nicht phototrophe Endoymbionten sind die Bakterien des Zwergtintenfisches Euprymna scolopes (Abb. 2A). Durch Jungtiere aus der Nahrung aufgenommene Aliivibrio fischeri setzen sich zunächst in einem spezifischen Organ gegenüber anderen Mikroorganismen durch, bevor sie in das Hautgewebe des Unterbauchs des Zwergtintenfisches eingelagert werden, um dort das Mondlicht zu imitieren und nachts die Jagd zu erleichtern.

In Schmierläusen wird ein verschachtelter Endosymbiontenkomplex maternal vertikal vererbt, so auch in Planococcus citri (Abb. 2B). Hier trägt das Betaproteobakterium Tremblaya selbst das endosymbiontische Gammaproteobakterium Moranella. Durch die Reduktion des Tremblaya-Genoms auf nur etwa 120 Gene ist dieses Bakterium metabolisch praktisch inaktiv, gemeinsam aber synthetisiert der Komplex Aminosäuren für den Wirt, der, durch die Nutzung eines Genrepertoires horizontalen Ursprungs, die Bausteine für die Peptidoglycanschicht des Betaproteobakterium liefert. Diese Matrjoschka-ähnliche Endosymbiose ist ein Musterbeispiel für die zellbiologische, biochemische und genetischen Verstrickung von Endosymbiont und Wirt, welche, wenn sie sich evolutionär stabilisiert, neue „chimäre“ Taxa hervorbringen.

\section{Stickstofffixierende Cyanobakterien: auf dem Weg zum Nitrogenosom}

Die ausreichende Stickstoffversorgung ist für Pflanzen und somit insbesondere für die Landwirtschaft ein Thema. Die Herstellung von Düngemitteln aber gehört zu einem der energieaufwendigsten Prozesse der Industrie. Zur Herstellung von einem Kilogramm fixiertem $\mathrm{N}_{2}$ in Form von Ammoniak oder
Ammoniumnitrat werden etwa 8,5 kWh verbraucht. Nur wenige Prozesse, wie die Verhüttung von Aluminium $(15,7 \mathrm{kWh} / \mathrm{kg})$, benötigen mehr.

Einige Eukaryoten profitieren von $\mathrm{N}_{2}$-fixierenden (Endo-)Symbionten. Neben den gut untersuchten rhizobienbildenen Leguminosen oder der endophytischen Interaktion mit diazotrophen Bakterien vieler Getreide [6], sind $\mathrm{N}_{2}$-fixierende Cyanobakterien, wie die der Diatomeen Epithemia turgida und Rhopalodia gibba oder des Wasserfarns Azolla (Abb. 2C), biologisch sehr interessant. Ihre Genome sind signifikant reduziert (um die $30 \%$ ), ihre Abhängigkeit ist permanent und sie werden vertikal vererbt. In jeder Spore des Farns finden sich wenige Cyanobakterien, die bei Keimung das Signal zur Differenzierung bekommen, sich vermehren und Heterozysten ausbilden, wobei die molekularen Details der Interaktion ungeklärt sind. Diese Endosymbiose ist an die 100 Millionen Jahre alt und so effizient, dass Azolla seine Biomasse in wenigen Tagen verdoppeln kann. Vor etwa 50 Millionen Jahren war Azolla so erfolgreich, dass der Farn über 100.000 Jahre lang so viel $\mathrm{CO}_{2}$ fixierte, dass die Erde signifikant abkühlte und all das dank seines cyanobakteriellen Symbionten, welcher zu einem Nitrogenosom evolviert.

\section{Literatur}

[1] Preiner M, Igarashi K, Muchowska KB et al. (2020) A hydrogen-dependent geochemical analogue of primordial carbon and energy metabolism. Nat Ecol Evol 4: 534-542 [2] Gould SB (2018) Membrane and evolution. Curr Biol 28: R367-R420

[3] Lane N, Martin WF (2010) The energetics of genome complexity. Nature 467: 929-934

[4] Knopp M, Garg SG, Handrich M, Gould SB (2020) Major changes in plastid protein import and the origin of the Chloroplastida. iScience 23: 100896

[5] Husnik F, Tashyreva D, Boscaro V et al. (2021) Bacterial and archaeal symbioses with protists. Curr Biol 31: R862R877

[6] Coba de la Peña T, Fedorova E, Pueyo JJ, Lucas MM (2018) The symbiosome: legume and rhizobia co-evolution toward a nitrogen-fixing organelle? Front Plant Sci 8: 2229

Funding note: Open Access funding enabled and organized by Projekt DEAL. Open Access: Dieser Artikel wird unter der Creative Commons Namensnennu 4.0 International Lizenz veröffentlicht, welche die Nutzung, Vervielfältigung, Bearbeitung, Verbreitung und Wiedergabe in jeglichem Medium und Form erlaubt, sofern Sie den/die ursprünglichen Autor(en) und die Quelle ordnungsgemäß nennen, einen Link zur Creative Commons Lizenz beif
angeben, ob Änderungen vorgenommen wurden. Die in diesem Artikel angeben, ob Anderungen vorgenommen wurden. Die in diesem Artikel
enthaltenen Bilder und sonstiges Drittmaterial unterliegen ebenfalls der enthaltenen Bilder und sonstiges Drittmaterial unterliegen ebenfalls der
genannten Creative Commons Lizenz, sofern sich aus der Abbildungslegende genannten Creative Commons Lizenz, sofern sich aus der Abbildungsleg
nichts anderes ergibt. Sofern das betreffende Material nicht unter der genannten Creative Commons Lizenz steht und die betreffende Handlung nicht nach gesetzlichen Vorschriften erlaubt ist, ist für die oben aufgeführten Weiterverwendungen des Materials die Einwilligung des jeweiligen Rechteinhabers einzuholen. Weitere Details zur Lizenz entnehmen Sie bitte der Lizenzinformation auf http://creativecommons.org/licenses/by/4.0/deed.de.

Korrespondenzadresse:

PD Dr. Sven B Gould

Institut für Molekulare Evolution

Heinrich-Heine-Universität Düsseldorf

Universitätsstraße 1

D-40225 Düsseldorf

gould@hhu.de

www.cellevo.de

Mona Schreiber
Jahrgang 1985. Studium der (biologischen) Anthropologie und der deutschen Philo-
logie an der Universität Mainz. 2014 Magister zur Domestikation des Hausrindes.
Doktorandin am Leibniz Institut für Pflanzengenetik und Kulturpflanzenforschung (IPK)
in Gatersleben. 2019 Promotion über die Domestikation von Getreide. 2019-2020
Postdoc am IPK. Seit 2021 Postdoc in der Pflanzenzellbiologie an der Universität
Marburg.
Sven B. Gould
Jahrgang 1976. 2006 Promotion über den Proteintransport in komplexen Plastiden,
im Anschluss Postdoc an der Universität Melbourne, Australien, zur Cytoskelett-
evolution. Seit 2010 an der Universität Düsseldorf, dort erst Nachwuchsgruppenleiter,
dann Habilitation im Jahr 2015 über die Funktion und Evolution eukaryotischer
Kompartimente und 2019 apl. Professur. Aktuell zusätzlich Vertretungsprofessor an
der Universität Marburg für Zellbiologie der Pflanzen.

